\title{
Prevalence \& Natural History of Tricuspid Regurgitation in Ischemic Mitral Regurgitation Patients
}

\author{
Ofir Koren ( $\nabla$ drkorenofir@gmail.com ) \\ Emek Medical Center \\ Henda Darawsha \\ Emek Medical Center \\ Ehud Rozner \\ Emek Medical Center \\ Yoav Turgeman \\ Emek Medical Center
}

\section{Research Article}

Keywords: Mitral regurgitation, Ischemic Mitral regurgitation, Tricuspid regurgitation, Echocardiography, Heart Failure

Posted Date: December 21st, 2020

DOl: https://doi.org/10.21203/rs.3.rs-128018/v1

License: (9) This work is licensed under a Creative Commons Attribution 4.0 International License. Read Full License

Version of Record: A version of this preprint was published at BMC Cardiovascular Disorders on April 21st, 2021. See the published version at https://doi.org/10.1186/s12872-021-01982-y. 


\section{Abstract}

\section{Background}

Functional tricuspid regurgitation (FTR) is most often secondary to left-sided heart pathology involving the mitral valve. We studied the incidence, clinical impact, risk factors, and natural history of patients who developed FTR due to an ischemic cause of mitral regurgitation (IMR). We conducted a retrospective cohort study based on data collected from January 2012 to December 2014. Patients diagnosed with IMR were eligible for the study. The mean follow-up was five years.

\section{RESULTS}

The study group consisted of 134 patients with IMR divided into two groups based on FTR development (FTR vs non-FTR group). Forty patients were diagnosed with FTR (30.1\%). FTR patients were older (63.0 \pm 10.4 vs $57.1 \pm 11.0$, respectively, $\mathrm{p}<0.05)$ with a high incidence of previous coronary artery disease $(p<.006)$. Severe IMR, high pulmonary arterial pressure (PAP), and failed revascularization were significant predictors of FTR development $(p<.001, p<.005, p<.003$, respectively). Low systolic left ventricle function was a predictor for FTR progression.

\section{CONCLUSION}

Clinical observations showed that FTR development due to IMR is common. Elderly patients with ischemic heart disease are at particularly high risk. FTR incidence and severity are directly proportional to the severity of IMR. FTR tends to deteriorate in about one-fifth of IMR patients and is mainly affected by the left ventricular systolic function.

\section{Introduction}

Acute myocardial infarction directly affects myocardium viability and functionality and leads to structural remodeling and conduction changes seen immediately after an injury. The remodeling process results in valvular dysfunction and eventually hemodynamic changes. Ischemic mitral regurgitation (IMR) is a complication observed following the remodeling of the injured left ventricle. ${ }^{1-3}$

IMR is defined as mitral regurgitation following structural remodeling due to ischemic myocardial injury that is not directly related to valvular or sub-valvular pathologies such as mitral valve prolapse, endocarditis, autoimmune disease, medication, or direct radiation effect. The mechanism of IMR seems to be limited to leaflet motion, mainly due to shortening and limited relaxation of the ischemic papillary muscles. IMR is often classified as type IIIb of the Carpentier classification introduced in 1983, involving a restricted leaflet motion, most commonly the posterior leaflet. ${ }^{4}$ There can also be a widening of the annulus diameter of the failing LV and secondary leaflet malcoaptation. Ischemic mitral regurgitation may develop after myocardial infarction in $11-59 \%$ of patients. ${ }^{5-7}$ 
Functional tricuspid regurgitation (FTR) is the consequence of various structural and functional changes involving the tricuspid annulus and the right ventricle. It is mostly secondary to left-sided mitral pathology in the setting of mitral regurgitation, mitral stenosis, and aortic stenosis. ${ }^{8-10}$ patients with FTR and IMR have a three-fold higher risk of developing heart failure and double the risk of mortality. ${ }^{11-16}$ The prevalence of tricuspid regurgitation in relation to mitral valve disease was described in recent studies addressing general left-sided pathology. ${ }^{17-20}$ The prevalence and natural history of FTR in the setting of mitral regurgitation following myocardial infarction was less known.

\section{Methods}

\section{PLANNING OF THE STUDY}

We conducted an observational cohort study using the computerized database of Emek Medical Center, a university-based secondary care center. Information regarding patient characteristics, demographic, and angiographic details were collected and analyzed using the Clalit health service database. Patients enrolled in the study were admitted to our heart institute for acute myocardial infarction from January 2012 to December 2014. Patients with primary mitral and tricuspid pathology and secondary pulmonaryrelated tricuspid regurgitation were excluded. The study population consisted of patients who developed mitral regurgitation following myocardial infarction. Patients were eligible for the study provided they met the inclusion criteria (Table 1s-supplementary) and had at least one transthoracic echocardiogram (TTE) at the time of hospitalization and at least one TTE during follow-up with no new Mls. The mean follow-up time was five years.

\section{ECHOCARDIOGRAPHIC ANALYSIS}

All echocardiograms were performed at the time of hospitalization using General Electric VIVID-75 s, VIVID 95, Philips CVx, and a portable bedside VIVID-1. The echocardiographic reports were prospectively reviewed independently by senior cardiologists using the Carestream Vue imaging system. In case of disagreement, an additional certified cardiologist was consulted. Echocardiographic images were assessed based on mitral and tricuspid insufficiency using an integrated approach taking into consideration valvular morphology, width of the proximal jet, color Doppler flow, jet area relative to the atrium, and hepatic and pulmonary flow patterns. LV ejection fraction (LVEF) was visually assessed (eyeball) in multiple acoustic windows and calculated using the biplane Simpson method according to the American Society of Echocardiography guidelines 2017. Systolic pulmonary artery pressures were calculated as the sum of the peak tricuspid regurgitation systolic gradient and the estimated vena cava central pressure (taking into consideration size and respiratory variation).

\section{SAMPLE SIZE}

The planned sample size is based on multiple variables such as IMR and FTR prevalence and a group difference of at least about $15 \%$ to demonstrate a statistical difference of $80 \%$ and alpha of $5 \%$ in a twosided test. We had to include a minimum sample size of 164 patients. Preliminary analysis reveals that 
one out of five patients will be eligible for the study. Following these analyzes, we extended the duration of recruitment for two years.

\section{ETHICS}

The study was approved by Emek Medical Center Ethics Committee following the Helsinki Convention No 0105 - 17 EMC. Informed consent was waived due to the use of anonymous patient data and the retrospective nature of the study.

\section{STATISTICAL ANALYSIS}

A chi-square test was performed to analyze the association between the study groups and categorical variables. For continuous variables, we used the t-test (alternatively the Wilcoxon two-sample test). Categorical variables were presented using frequencies and percentages. Continuous variables were presented using mean \pm standard deviation. All confidence intervals are at the $95 \%$ level. TR progression was analyzed using the Mantel-Cox multivariant analysis and R-squared analysis. Multivariable models and logistic regression analysis were used to estimate the predictors of FTR. Differences considered statistically significant were at the 2 -sided $p$ level of 0.05 . The statistical analyses were performed using SAS 9.4 software. A p-value $<0.05$ was considered significant.

\section{Results Of The Study}

During the study period, 960 patients were admitted to the intensive cardiac unit due to acute Ml. Of these, 275 patients were excluded from the study: 260 patients due to non-ischemic originating mitral regurgitation or previous known mitral regurgitation, five patients due to primary mitral disease (two due to rheumatic heart disease, two due to mitral valve prolapse and one due to chest radiation), and nine patients due to secondary non-IMR-related tricuspid pathology.

Of the 685 eligible patients, 175 (25.6\%) developed ischemic mitral regurgitation. Thirty-five patients were excluded due to a lack of adequate follow-up TTE exams and poor imaging quality. Thus, 134 patients were in the final study cohort (Fig. 1).

Forty patients (29\%, 95\% Cl: 22.75-38.07\%) developed functional tricuspid regurgitation (FTR) during follow-up. Demographic characteristics of the two groups revealed that patients who developed FTR were older $(63.0 \pm 10.4$ and $57.1 \pm 11.0$, respectively, $p<0.05)$ with a high incidence of vascular disease either coronary $(p<0.006)$ or peripheral $(p<0.009)$. Failed coronary revascularization was a strong positive predictor of FTR development $(p<.003)$. No gender difference was found between the groups. Smoking, hyperlipidemia, diabetes, and chronic kidney failure were not predictive of FTR development (Table 1). 
Table 1

Baseline Characteristics

\begin{tabular}{|c|c|c|c|}
\hline Characteristic & $\begin{array}{l}\text { FTR } \\
(n=40)\end{array}$ & $\begin{array}{l}\text { No-FTR } \\
(n=94)\end{array}$ & p-Value \\
\hline Age & $\begin{array}{l}63.0 \pm 10.4 \\
(62.5 ; 45-85)\end{array}$ & $\begin{array}{l}57.1 \pm 11.0 \\
(57 ; 34-89)\end{array}$ & .005 \\
\hline Male & $30(75.0)$ & $76(80.9)$ & .45 \\
\hline Female & $10(25.0)$ & $18(19.1)$ & \\
\hline Smoking & $16(40.0)$ & $59(62.8)$ & .02 \\
\hline Chronic Kidney Failure & $3(7.5)$ & $3(3.2)$ & .36 \\
\hline Diabetes (types 1 and 2) & $14(35.0)$ & $38(40.4)$ & .56 \\
\hline Previous Coronary Catheterization & $20(50.0)$ & $23(24.5)$ & 004. \\
\hline Previous Bypass Surgery & $4(10.0)$ & $3(3.2)$ & 20. \\
\hline Peripheral vascular disease & $5(12.5)$ & $1(1.1)$ & .009 \\
\hline Atrial fibrillation & $4(10.0)$ & $3(3.2)$ & .20 \\
\hline STEMI & $39(97.5)$ & $84(90.4)$ & 17. \\
\hline \multicolumn{4}{|l|}{ KILLIP Score } \\
\hline 1 & $0(0.0)$ & $0(0.0)$ & 16. \\
\hline 2 & $32(80.0)$ & 83 (88.3) & \\
\hline 3 & $1(2.5)$ & $0(0.0)$ & \\
\hline 4 & $4(10.0)$ & $3(3.2)$ & \\
\hline New Q on ECG & $8(20.0)$ & 11(11.8) & 22. \\
\hline Infarct Area & & & .39 \\
\hline Anterior & $20(51.3)$ & $42(44.7)$ & \\
\hline Inferior & $15(38.5)$ & $37(39.4)$ & \\
\hline Lateral & $1(2.6)$ & $4(4.3)$ & \\
\hline Posterior & $1(2.6)$ & $0(0.0)$ & \\
\hline Other & $2(5.1)$ & $11(11.7)$ & \\
\hline
\end{tabular}

$\Psi$ Defined as failed to perform balloon dilatation or stent implantation $\mathrm{PCl}$, percutaneous coronary intervention 


\begin{tabular}{|c|c|c|c|}
\hline Characteristic & $\begin{array}{l}\text { FTR } \\
(n=40)\end{array}$ & $\begin{array}{l}\text { No-FTR } \\
(n=94)\end{array}$ & p-Value \\
\hline Failed PCl ${ }^{*}$ & $5(12.5)$ & $4(4.3)$ & .03 \\
\hline Complications after $\mathrm{PCl}$ & $6(15.0)$ & $6(6.5)$ & .18 \\
\hline \multicolumn{4}{|l|}{ Culprit artery } \\
\hline LAD & $65(48.5)$ & $20(50.0)$ & $45(47.9)$ \\
\hline $\mathrm{RCA}$ & $45(33.6)$ & $14(31.1)$ & $31(33.0)$ \\
\hline $\mathrm{CX}$ & $5(3.7)$ & $3(7.5)$ & $2(2.1)$ \\
\hline Other & 19() & $3(7.5)$ & $16(17.0)$ \\
\hline \multicolumn{4}{|c|}{$\Psi$ Defined as failed to perform balloon dilatation or stent implantation } \\
\hline $\mathrm{PCl}$, percutaneous corona & tion & & \\
\hline
\end{tabular}

Most patients who developed IMR had STEMI (92\%). The ST-segment elevation on ECG, KILLIP class, and infarct territory was not associated with FTR development or progression (Table 1). We did not find a significant statistical correlation between the infarct-related artery involved and the development of FTR. Our analysis did not cover the correlation of multivessel disease with the development of FTR. Pulmonary arterial pressure (mean) and left ventricular systolic function as estimated by ejection fraction appeared to have a significant effect on FTR progression ( $p<0.05, p<0.02$, respectively) but did not affect FTR development ( $p<0.5, p<0.54$, respectively) (Table $2 s$ and Table $3 \mathrm{~s}$ ). The distribution of incidence events and their severity among IMR patients appear to be directly and linearly linked to FTR development and progression (Fig. 2). During follow-up, the development of new FTR was observed in almost third of the patients. FTR progressed in one-fifth of patients within 1.67 years $(1.8 \pm$ SD). The development of FTR was strongly associated with elevated pulmonary arterial pressure and reduced left ventricular ejection fraction (LVEF) (Fig. 3). Atrial Fibrillation was found to be associated with TR severity $2(X 2=0.96, p$ $>$.62).

Patients who developed FTR had a lower overall survival rate than patients who did not have FTR. Multivariable models to predict FTR in IMR patients indicated a statistically significant difference in survival between the two groups at the time to progression to severe FTR (Mantel Cox $\chi 2=5.02, p<.025$ ). FTR group mean survival time was 4.58 years (95\% Cl: $4.11-5.04$ years) and the no-FTR group was 5.15 years (95\% Cl: $4.90-5.40$ years) (Fig. 4$)$.

FTR progression was associated with heart failure at admission. There were statistically significant greater odds of FTR progression among patients with heart failure compared to patients with no heart failure at admission (OR: 8.09; 95\% Cl: 3.30-19.89, p<.001). 


\section{Discussion}

The results of our study support recent reports of bivalvular deterioration in heart failure patients with reduced ejection fraction (HFrEF) and the interconnection between the mitral and tricuspid apparatuses. Our study highlights a specific group of patients with ischemic-related mitral regurgitation and its effect on the development of functional TR.

Multivalve involvement was described in the past regarding rheumatic heart disease and congenital heart disease and not extensively in the context of ischemic heart disease.

Myocardial infarction is still the most common cause of HFrEF and until recently it was thought that its major effect was directed toward left-sided pathology through an extensive remodeling process.

IMR is a common complication after myocardial infarction that requires adequate follow-up regardless of the appearance of clinical symptoms. Mild asymptomatic IMR usually tends to be neglected; however, our study demonstrates a linear relationship between the development of mitral regurgitation and the development of functional tricuspid regurgitation as a result of myocardial infarction. This linear connection, we believe, seems to involve volume and pressure overload, shifting from the left to the right side through the pulmonary vasculature.

Nearly one-third of IMR patients developed FTR during the five-year follow-up period. Elderly patients with extensive myocardial damage were at particularly high risk. Almost one-fifth of patients who developed IMR and LV dysfunction had a significantly high risk of FTR deterioration.

Bivalvular regurgitation, particularly with involvement of the tricuspid valve, greatly influences the prognosis of heart failure, limits therapeutic options, and substantially increases the overall mortality.

IMR should be properly monitored and followed up regardless of symptoms. FTR should be assessed annually to avoid significant deterioration and to start prompt treatment for heart failure before the first signs of heart failure appear.

\section{Limitation Of The Study}

Our study is designed in a retrospective fashion. The data were analyzed retrospectively based on computerized patient medical records. Echocardiographic images were assessed according to the ASE criteria.

Quantification of the severity of mitral and tricuspid regurgitation was based on qualitative grading (visual assessment) using parameters such as color flow, jet area; CWD jet; LV and RV size, function, and volume; PA pressure; MV and TV morphology; and LA and RA size. These assessment methods may be confounded by several technical and hemodynamic factors, including the eccentricity of the jets and atrial size. However, we cannot accurately apply quantitative or semiquantitative methods because this is a retrospective study. 
The influence of heart failure medical therapy and its contribution to valvular dysfunction could not be evaluated here. Larger samples are needed to evaluate these factors.

\section{Conclusion}

One-third of patients who develop ischemic mitral regurgitation (IMR) will develop functional tricuspid regurgitation (FTR) at a five-year follow-up. The incidence and severity of FTR are highly influenced by the severity of the IMR. Elderly patients with ischemic heart disease who had extensive myocardial damage and for whom revascularization failed are at high risk of IMR and FTR development. LV function significantly influences FTR deterioration.

\section{Declarations}

\section{Ethics approval and consent to participate}

- The study was approved by Emek Medical Center Ethics Committee in accordance with the Helsinki Convention. Informed consent was waived due to the confidentiality of the patient data and the methodology of the study.

\section{Consent for publication}

$-N / A$

\section{Availability of data and materials}

- The datasets used and/or analyzed during the current study are available from the corresponding author on reasonable request.

\section{Competing interests}

- The authors declare that they have no competing interests.

\section{Funding}

- The authors have not declared a specific grant for this research from any funding agency in the public, commercial, or not-for-profit sectors.

\section{Authors' contributions}


- OK, HD, and ER contributed to the writing, editing, formatting of the main manuscript, and production of the figures. ER, OK, and YT provided care to the patients and revised the manuscript. OK certified that all authors have contributed and met the criteria for authorship.

\section{Acknowledgment}

- We would like to thank the echocardiographic technicians Shirin Huri and Limor Shalom for their significant contribution to the research.

\section{Authors' information (optional)}

$-\mathrm{N} / \mathrm{A}$

\section{References}

1. Ziegler, Kimberly; Quillen, Terrilynn Fox (2005): Mitral valve regurgitation after myocardial infarction. Nursing 35 (11), S. 88

2. Birnbaum, Yochai; Chamoun, Antonio J.; Conti, Vincent R.; Uretsky, Barry F. (2002): Mitral regurgitation following acute myocardial infarction. Coronary artery disease 13 (6), S. 337-344.

3. Bursi, Francesca; Enriquez-Sarano, Maurice; Jacobsen, Steven J.; Roger, Veronique L. (2006): Mitral regurgitation after myocardial infarction: a review. The American journal of medicine 119 (2), S. 103-112.

4. Carpentier A. Cardiac valve surgery-the "French correction". J Thorac Cardiovasc Surg. 1983 Sep;86(3):323-37. PMID: 6887954.

5. Bursi, Francesca; Enriquez-Sarano, Maurice; Nkomo, Vuyisile T.; Jacobsen, Steven J.; Weston, Susan A.; Meverden, Ryan A.; Roger, Veronique L. (2005): Heart failure and death after myocardial infarction in the community: the emerging role of mitral regurgitation. Circulation 111 (3), S. 295-301.

6. Kaul, S.; Spotnitz, W. D.; Glasheen, W. P.; Touchstone, D. A. (1991): Mechanism of ischemic mitral regurgitation. An experimental evaluation. Circulation 84 (5), S. 2167-2180.

7. Otsuji, Yutaka; Levine, Robert A.; Takeuchi, Masaaki; Sakata, Ryuzo; Tei, Chuwa (2008): Mechanism of ischemic mitral regurgitation. Journal of cardiology 51 (3), S. 145-156.

8. Ubago JL, Figueroa A, Ochoteco A, Colman T, Duran RM, Duran CG. Analysis of the amount of tricuspid valve anular dilation required to produce functional tricuspid regurgitation. Am J Cardiol 1983;52: 155-8.

9. Dreyfus GD, Martin RP, Chan KM, Dulguerov F, Alexandrescu C. Functional tricuspid regurgitation: a need to revise our understanding. J Am Coll Cardiol. 2015 Jun 2;65(21):2331-6. doi: 10.1016/j.jacc.2015.04.011. PMID: 26022823. 
10. Grigioni, Francesco; Detaint, Delphine; Avierinos, Jean-Francois; Scott, Christopher; Tajik, Jamil; Enriquez-Sarano, Maurice (2005): Contribution of ischemic mitral regurgitation to congestive heart failure after myocardial infarction. Journal of the American College of Cardiology 45 (2), S. 260-267.

11. Hillis, Graham S.; Moller, Jacob E.; Pellikka, Patricia A.; Bell, Malcolm R.; Casaclang-Verzosa, Grace C.; Oh, Jae K. (2005): Prognostic significance of echocardiographically defined mitral regurgitation early after acute myocardial infarction. American heart journal 150 (6), S. 1268-1275.

12. Perez de Isla, Leopoldo; Zamorano, Jose; Martinez Quesada, Mar; Corros, Cecilia; Ortiz, Pilar; Almeria, Carlos et al. (2005): Prognostic significance of ischemic mitral regurgitation after non-Q-wave acute myocardial infarction. The Journal of heart valve disease 14 (6), S. 742-748.

13. Salukhe, Tushar V.; Henein, Michael Y.; Sutton, Richard (2005): Ischemic mitral regurgitation and its related risk after myocardial infarction. Circulation 111 (3), S. 254-256.

14. Aronson, Doron; Goldsher, Noa; Zukermann, Robert; Kapeliovich, Michael; Lessick, Jonathan; Mutlak, Diab et al. (2006): Ischemic mitral regurgitation and risk of heart failure after myocardial infarction. Archives of internal medicine 166 (21), S. 2362-2368.

15. Soleimani, Mehrdad; Khazalpour, Michael; Cheng, Guangming; Zhang, Zhihong; Acevedo-Bolton, Gabriel; Saloner, David A. et al. (2011): Moderate mitral regurgitation accelerates left ventricular remodeling after posterolateral myocardial infarction. The Annals of thoracic surgery 92 (5), S. 1614-1620.

16. Sagie A, Freitas N, Chen MH, Marshall JE, Weyman AE, Levine RA. Echocardiographic assessment of mitral stenosis and its associated valvular lesions in 205 patients and lack of association with mitral valve prolapse. J Am Soc Echocardiogr 1997;10:141- 8.

17. Boyaci A, Gokce V, Topaloglu S, Korkmaz S, Goksel S. Outcome of significant functional tricuspid regurgitation late after mitral valve replacement for predominant rheumatic mitral stenosis. Angiology 2007;58:336-42.

18. Lee C-H, Laurence DW, Ross CJ, Kramer KE, Babu AR, Johnson EL, et al. Mechanics of the Tricuspid Valve-From Clinical Diagnosis/Treatment, In-Vivo and In-Vitro Investigations, to Patient-Specific Biomechanical Modeling. Bioeng (Basel, Switzerland). 2019 May;6(2).

19. Bartko PE, Arfsten H, Heitzinger G, Pavo N, Winter MP, Toma A, Strunk G, Hengstenberg C, Hülsmann M, Goliasch G. Natural history of bivalvular functional regurgitation. Eur Heart J Cardiovasc Imaging. 2019 May 1;20(5):565-573. doi: 10.1093/ehjci/jey178. PMID: 30508183.

20. Mutlak D, Khalii J, Lessick J, Kehat I, Agmon Y, Aronson D. Risk Factors for the Development of Functional Tricuspid Regurgitation and Their Population-Attributable Fractions. JACC Cardiovasc Imaging. 2020 Aug;13(8):1643-1651. doi: 10.1016/j.jcmg.2020.01.015. Epub 2020 Apr 15. PMID: 32305485 .

\section{Figures}




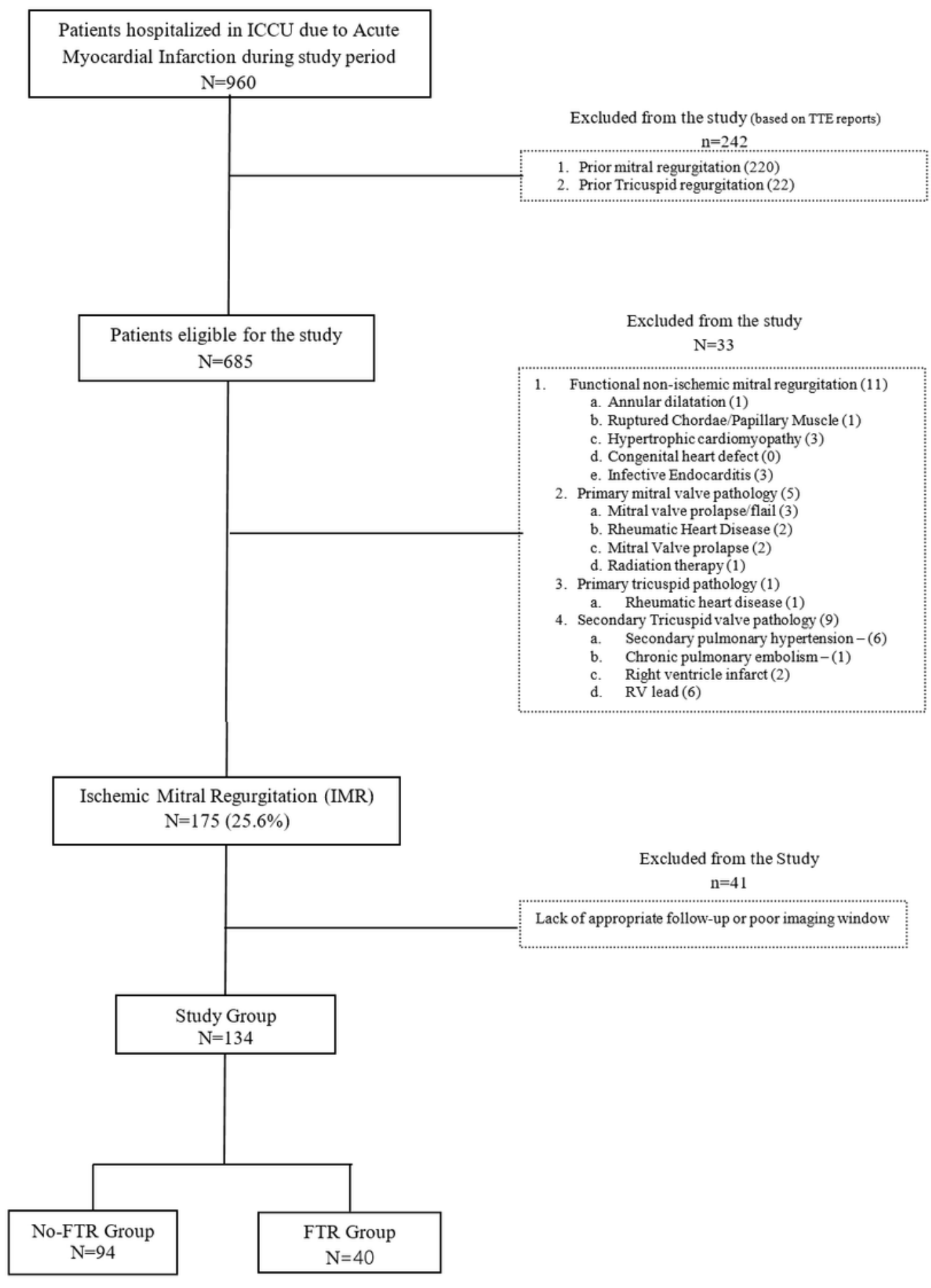

\section{Figure 1}

\section{Study design}


Distribution of prevalence and severity of FTR and IMR among study population

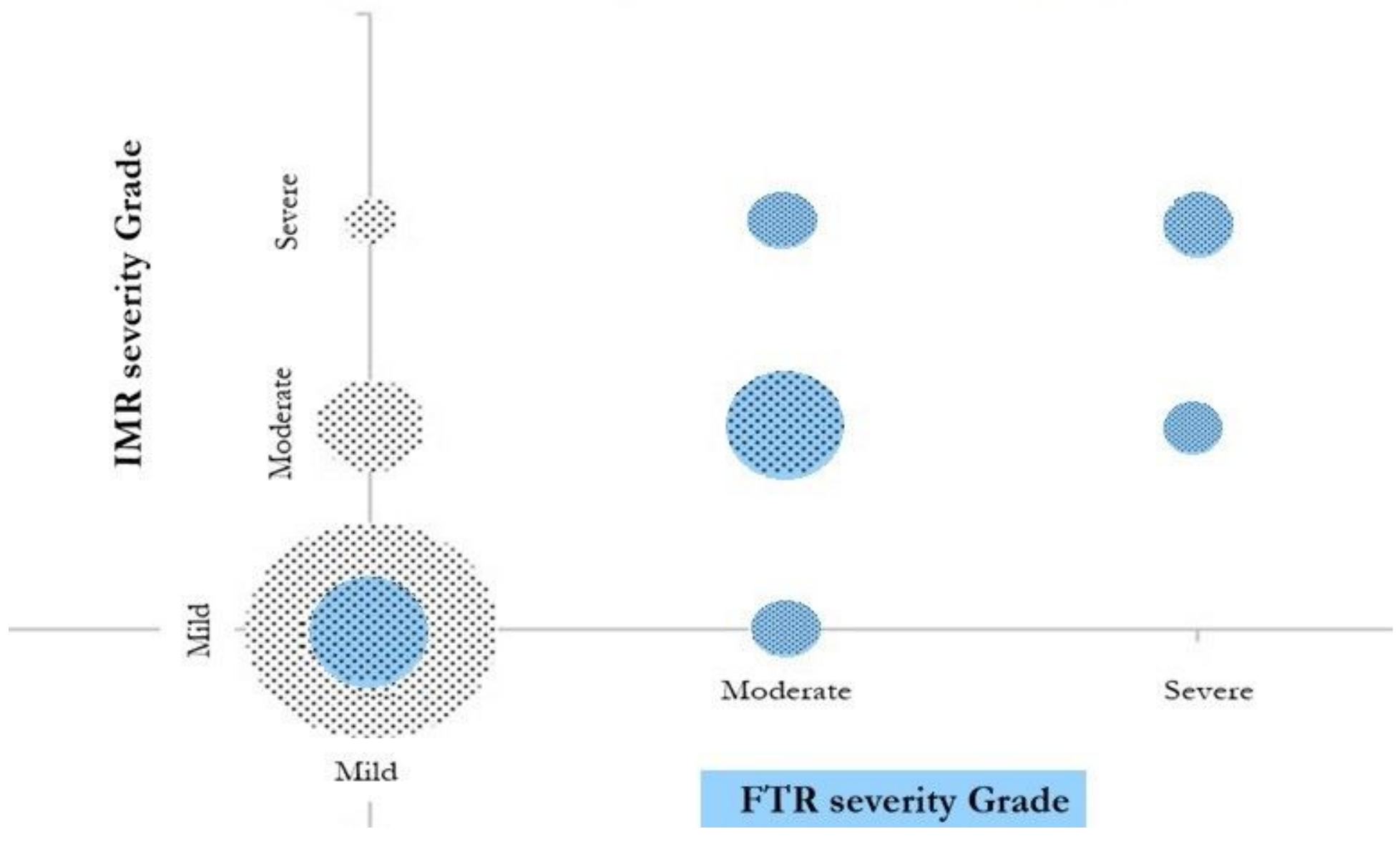

Figure 2

Distribution of prevalence and severity of valvular at indexed event 


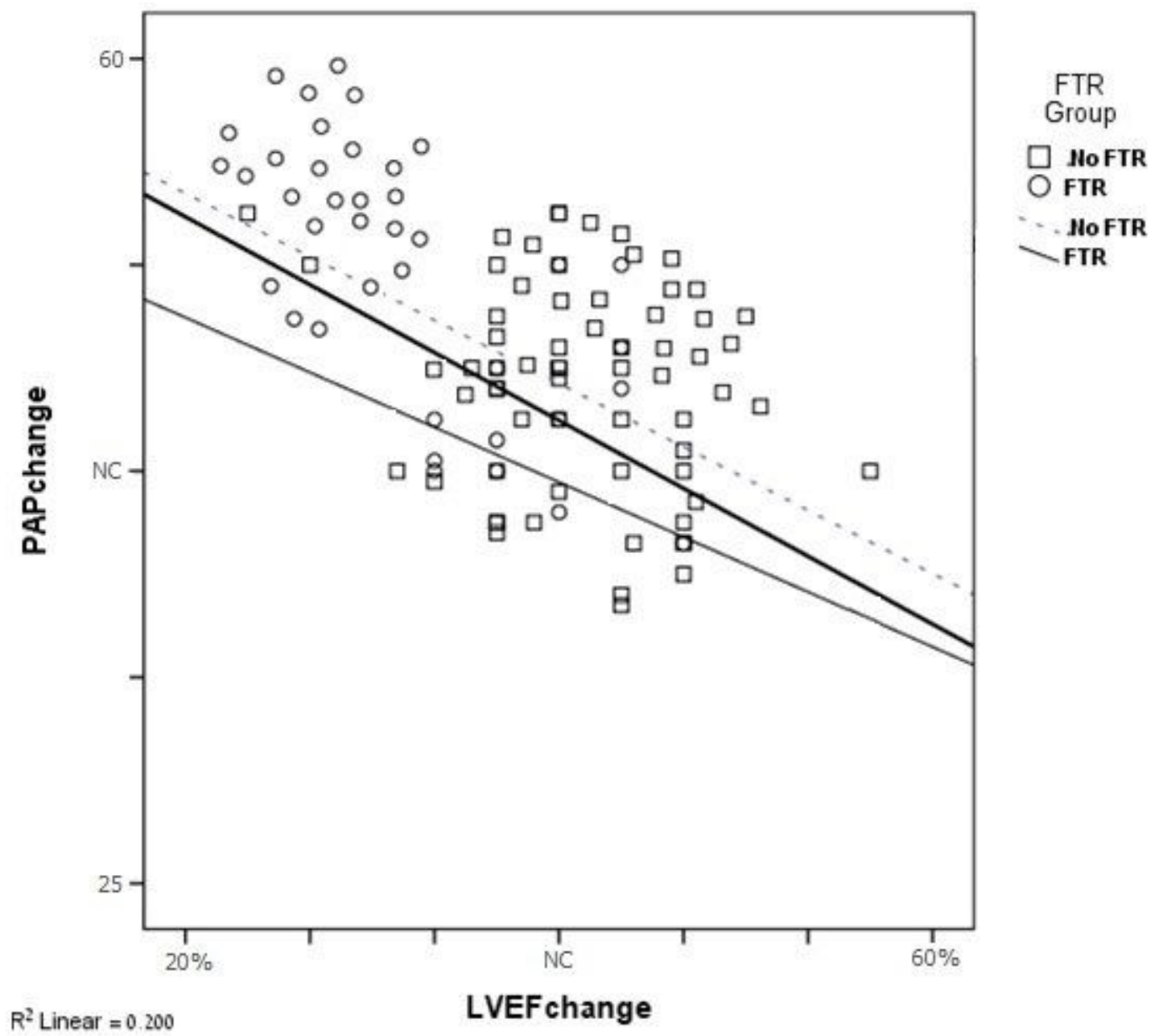

Figure 3

Distribution of FTR in relation to mean pulmonary arterial pressure and left ventricular ejection fraction progression 


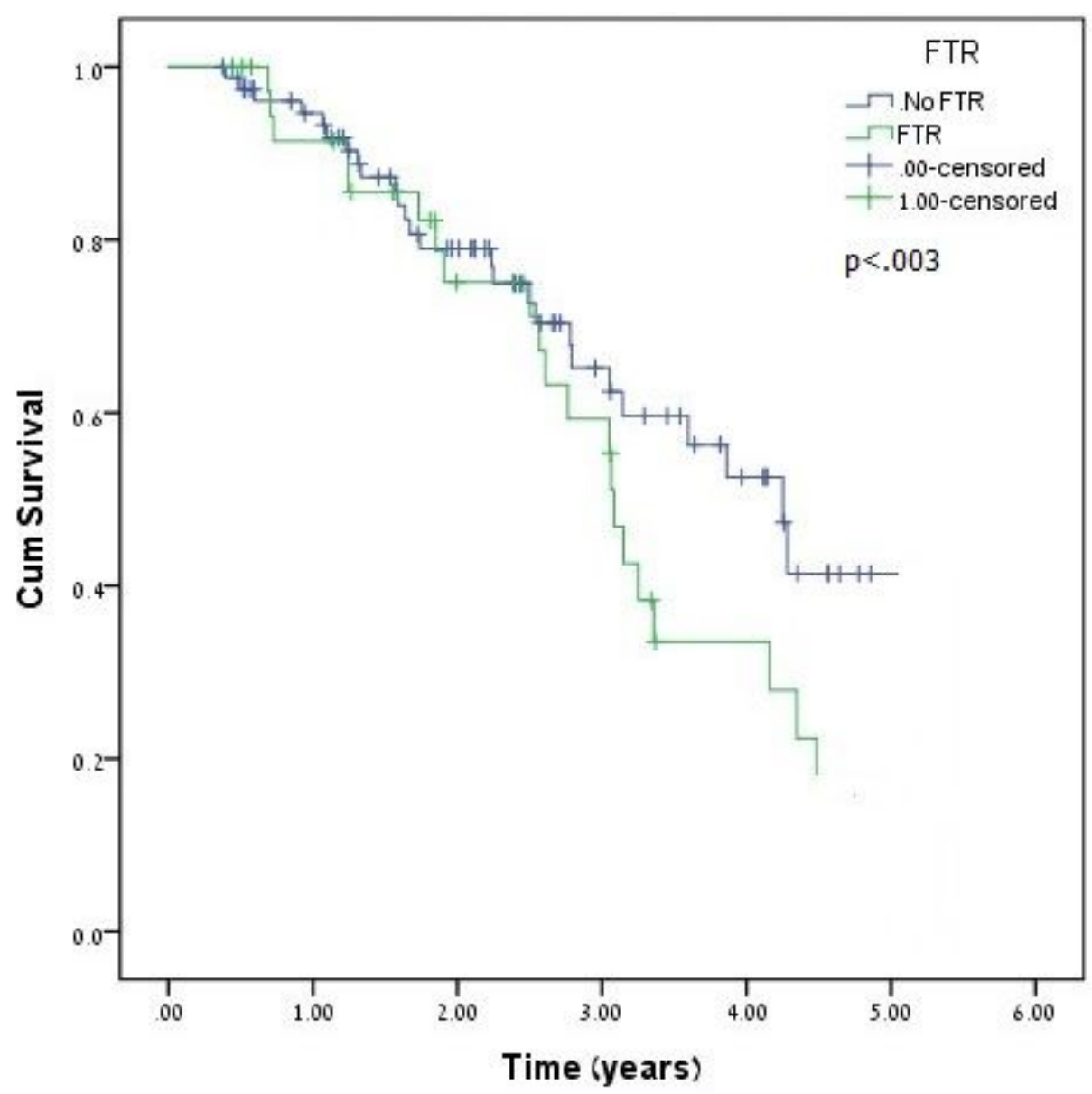

Figure 4

Kaplan-Mayer survival curve of FTR progression in IMR

\section{Supplementary Files}

This is a list of supplementary files associated with this preprint. Click to download.

- Supplementary.docx 